J Ästhet Chir 2022 - 15:1-2

https://doi.org/10.1007/s12631-021-00284-8

Angenommen: 24. November 2021

(C) The Author(s), under exclusive licence to Springer Medizin Verlag GmbH, ein Teil von Springer Nature 2021

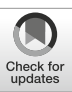

\section{Anatomie von Gesicht und Körper}

\author{
Sebastian Cotofana' $\cdot$ Konstantin Frank ${ }^{2}$ \\ ${ }^{1}$ Department of Clinical Anatomy, Mayo Clinic College of Medicine and Science, Rochester, USA \\ ${ }^{2}$ Abteilung für Hand-, Plastische und Ästhetische Chirurgie, Klinikum der Ludwig-Maximilian-Universität \\ München (LMU), München, Deutschland
}

Anatomie, ein Wort, das „auseinander schneiden" bedeutet, hat als naturwissenschaftliches Fach die heutige Medizin wie kein anderes Fach geprägt und beeinflusst. In den Anatomievorlesungen wird Medizin nicht selten als angewandte Anatomie beschrieben, um dieser leider aussterbenden Domäne doch noch ein wenig Hoffnung zu verleihen, bevor sie gänzlich von den klinischen Fächern verschluckt und inkorporiert wird. Die Frage, die ich mir als Anatom heutzutage stelle, ist: „Wie konnte es so weit kommen? Wie konnte die Anatomie derart an Wichtigkeit verlieren, dass an vielen internationalen Universitäten und medizinischen Lehreinrichtungen es gar kein Institut für Anatomie mehr gibt?" Die Antwort darauf ist entweder eine Verleugnung der realen Tatsachen, dass dem nicht so sei, oder, dass die Anatomie sich in der Vergangenheit zu wichtig genommen hat und sich langsam, aber stetig von der klinischen Medizin entfernt hat. Wenn man als Anatom ehrlich und reflektiert genug ist, wird man zustimmen, dass $50 \%$ (oder sogar mehr) des anatomischen Curriculums nicht in der Klinik gebraucht werden. (Hierbei sollte angemerkt werden, dass der Verfasser dieses Editorials die unfallchirurgische Schule durchlaufen hat und beide Seiten kennt.) Ob es daran liegt, dass erfolgreiche Absolventen eines Medizinstudiums lieber in die Klinik gehen mit guten Gehältern und langfristigen Arbeitsverträgen, als in kalten Präparationssälen Fragen von unvorbereiteten Studenten zu beantworten, kann ebenfalls dazu geführt haben, dass die Kluft zwischen anatomischer Ausbildung und Klinik immer weiter gewachsen ist. Es können meiner persönlichen Meinung nach viele Gründe angeführt werden und Schuldige an den Pranger gestellt werden, aber eine Lösung wird dadurch auch nicht eher gefunden werden. Von wesentlich größerer Bedeutung hingegen ist, was getan werden muss, damit Anatomie erneut ein klinischer Wegweiser werden kann, der Klinikern die Kompetenz an die Hand gibt, um sicherer und effektiver den Patientenvertrag erfüllen zu können.

Der an Bedeutung gewinnende Bereich derästhetischen Medizin, der multidisziplinär sowohl in den chirurgischen und nichtchirurgischen Fächern angesiedelt ist, gilt oftmals als der Wilde Westen der Medizin, und nicht selten wird der Begriff „Ästhetik" als Unwort verwendet. Der wichtigste Grund hierfür ist meiner persönlichen Meinung nach die eminenzbasierte Herangehensweise an Therapieplanung und Durchführung anstatt einer evidenzbasierten, wie es in allen anderen Fachgebieten der Medizin der Fall ist. Nicht selten wird als Leitlinie die persönliche Meinung einer Prominenz aus den "social medias" verwendet, willentlich oder unwillentlich vergessend, dass auf der anderen Seite des Skalpells oder der Injektionsnadel sich ein Patient befindet. Dass Leitlinien größtenteils fehlen und dass es kaum Grundlagenforschung in diesem Bereich gibt, sollte nicht an den Behandlern ausgelassen werden. Denn es ist nicht deren primäre Aufgabe, dieses Wissen zu generieren, sondern es anzuwenden.

In den letzten Jahren hat glücklicherweise das Fachgebiet der Anatomie eine Renaissance erlebt, besonders wenn es um die Anatomie des Gesichts oder der suprafaszialen Weichteile des Körpers geht. Neue Erkenntnisse sind veröffentlicht worden, worunter die Biomechanik 
des Gesichts, die arterielle Durchblutung des Kopf- und Halsbereiches und das superfizielle fasziale System genannt werden müssen. Diese neuen Begebenheiten können die klinischen Fachkollegen leiten, und das publizierte Wissen kann in Leitlinien aufgearbeitet werden.

Im Folgenden sollen diese neuen Erkenntnisse im Einzelnen dargelegt und mit klinischer Erfahrung gepaart werden, um aufzuzeigen, dass weder die Anatomie am Aussterben ist, noch dass alle Hoffnung auf eine evidenzbasierte ästhetische Medizin verloren ist.

Sebastian Cotofana, MD, PhD, PhD

Dr. Konstantin Frank

\section{Korrespondenzadresse}

\section{Sebastian Cotofana, MD, PhD, PhD}

Department of Clinical Anatomy, Mayo Clinic College of Medicine and Science

Mayo Clinic, Stabile Building 9-38, 200 First

Street, 55905 Rochester, MN, USA

cotofana.sebastian@mayo.edu

Interessenkonflikt. S. Cotofana und K. Frank geben an, dass kein Interessenkonflikt besteht.

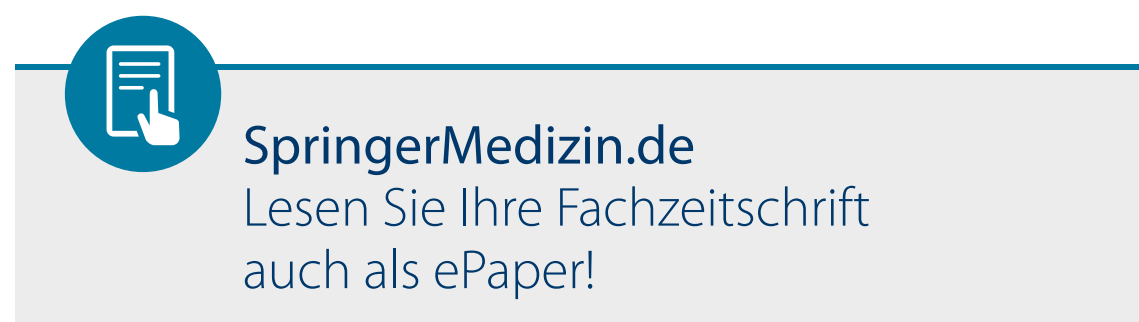

Als Abonnent können Sie Ihre Zeitschrift in verschiedenen Formaten lesen. Wählen Sie je nach Vorliebe und Situation aus, ob Sie die Zeitschrift als PrintAusgabe, in Form von einzelnen Beiträgen auf springermedizin.de oder aber als komplette, elektronische ePaper-Ausgabe lesen möchten.

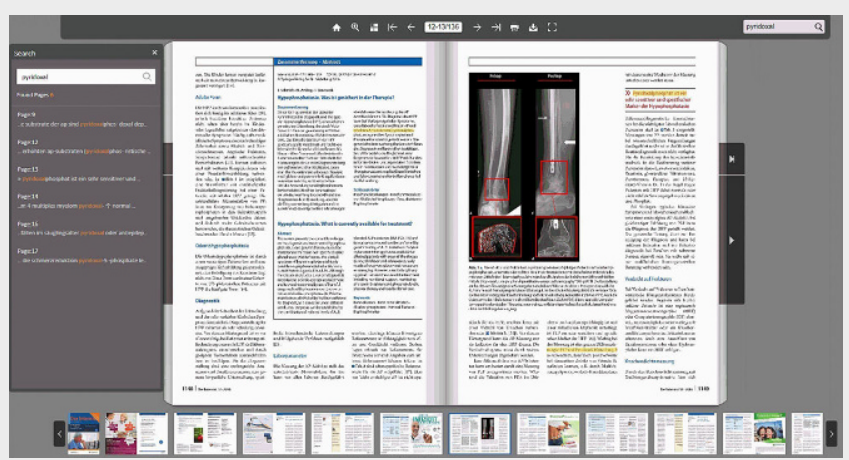

Die ePaper sind die identische Form der gedruckten Ausgaben. Sie sind nutzbar auf verschiedenen Endgeräten wie PC, Tablet oder Smartphone

Das sind die Vorteile des ePapers:

$>$ Das verlinkte Inhaltsverzeichnis führt Sie direkt zum gewünschten Beitrag.

$>$ Eine Suchfunktion ermöglicht das Auffinden von Schlagworten innerhalb der Zeitschrift.

$>$ Jede Ausgabe kann als PDF heruntergeladen und damit auch offline gelesen werden bzw. auch gespeichert oder ausgedruckt werden.

> Als Abonnent haben Sie Zugang zu allen ePaper-Ausgaben ab 2016.

Sie finden die ePaper auf SpringerMedizin.de bei der jeweiligen Ausgabe Ihrer Fachzeitschrift. Klicken Sie auf den Button „Ausgabe als ePaper lesen“.. 